\title{
Optimized Opportunistic Routing in Highly Dynamic Ad hoc Networks
}

\author{
Varun G Menon ${ }^{1 *}$ \\ ${ }^{1}$ SCMS School of Engineering and Technology, Kerala, India 683582, \\ Email: varunmenon@scmsgroup.org
}

\begin{abstract}
:
Opportunistic Routing is a promising paradigm that has been proposed for efficient and reliable transfer of data packets in mobile ad hoc networks. This routing strategy takes advantage of the broadcasting nature of the wireless medium to increase the number of probable forwarding devices and improves the reliability of data transfer in the network. Opportunistic Routing utilizes the reception of the same broadcasted packet at multiple devices in the network and selects one best forwarder dynamically from the set of multiple receivers. A number of opportunistic routing protocols have been proposed over these years for effective data delivery in ad hoc networks. But as the speed and mobility of devices increases in the network, performances of the existing opportunistic routing protocols degrade considerably, leading to reduced Quality of Service and poor transmission efficiency. The exceptional potential of opportunistic routing is thus underutilized. In this research we introduce Optimized Opportunistic Routing (OOR) strategy that guarantee excellent Quality of Service and high transmission efficiency to the latest applications using opportunistic routing for communication in highly dynamic ad hoc networks. Simulation results show that our method achieve significant performance improvements compared to all other existing opportunistic routing protocols in highly dynamic mobile ad hoc networks.
\end{abstract}

\section{Keywords}

Ad Hoc Networks; Highly Dynamic; Mobility; Opportunistic Routing; Performance Improvement; Transmission Inefficiency

\section{Introduction}

Mobile ad hoc networks (MANETs) [1-10] are a collection of wireless devices like mobile phones, laptops, PC's and iPads that can form instantaneous temporary networks without the support of any pre-existing network infrastructure or centralized control. It works as an autonomous system of mobile hosts connected by wireless communication links. The network is configured in a way that all the devices can dynamically join or leave the network at any time 
without disrupting communication between other devices. Every device in the network plays the dual role of a router and a host, cooperates and coordinates with each other to make routing decisions in the network. Data is transmitted in the network in a store and forward manner from the source node to the destination node via the intermediate nodes. Ease of deployment, speed of deployment and the ability to self-organize and self-adapt without the help of any underlying infrastructure has contributed to the growing popularity of MANETs in research as well as in industry. Today MANETs are used for communication and resource sharing in wide range of applications.

Numerous advancements in wireless technology have enabled mobile devices in MANETs to move freely with higher speeds in random directions. The mobility and speed of these wireless devices have become highly unpredictable and is increasing day by day. Also the number of connected devices in the network is increasing rapidly leading to highly dense and scalable ad hoc networks. These scenarios have led to the generation of highly dynamic mobile ad hoc networks (HDMANETs) in which numerous number of connected wireless devices move with higher speeds in random directions. HDMANETs offer a number of challenges to various applications due to its unique properties. The main characteristics of HDMANETs include continuous movement of wireless devices, higher speeds of wireless devices, unpredictable movement of devices in random directions, higher number of connected devices, dynamic connections, disconnections and reconnections of devices.

Routing and timely delivery of data packets have remained highly challenging task in HDMANETs because of the unpredictable movement and higher speeds of the connected devices. Traditional topology based protocols like Destination Sequenced Distance Vector (DSDV) [11], Optimized Link State Routing (OLSR) [12], Topology Dissemination Based on Reverse-Path Forwarding (TBRPF) [13], Dynamic Source Routing (DSR) [14], Associativity-Based Routing [15], Ad hoc On Demand Distance Vector (AODV) [16] and Temporally Ordered Routing Algorithm (TORA) [17] depend on predetermined routes between source and destination devices. With highly mobile nodes it is impossible to maintain a deterministic route. Also the discovery and recovery procedures are time and energy consuming. Thus they suffer from serious performance degradation in HDMANETs and are highly ineffective. The new class of protocols known as geographic routing protocols [18-22] used location information to route the packets in a hop by hop fashion from the source device to the destination device. Greedy Perimeter Stateless 
Routing (GPSR) [18] is one of the most referenced protocol in this category. This protocol selects the device that has maximum progress to the destination (nearest to the destination) as the best forwarder to forward the data packet. When this strategy was not possible in some region in the network, GPSR used a technique of routing around the perimeter of the region. But the major problem with this category of protocols in HDMANETs was when the best forwarder device moved away from the current location and was unable to forward the data packet.

A major breakthrough in this area was provided with the discovery of opportunistic routing (OR) and opportunistic data forwarding [23]. Opportunistic routing protocols [23-33] were proposed to offer reliable data delivery and excellent Quality of Service (QoS) to applications using MANETs for communication and resource sharing. Numerous advantages offered by OR protocols have enabled researchers to use them for communication in MANETs deployed in some of the harshest environments like volcanoes, hurricane affected regions and underground mines. They are currently being used in a wide range of applications spanning from communication between rescue workers in disaster recovery operations [34-36], battlefield communications [37], industrial sites interconnection [38], emergency evacuation and recovery [39, 40] setting up communication in conferences and exhibitions to providing internet connections in rural areas [41].

Today, these applications using opportunistic routing for communication in MANETs are faced with two major challenges; the exponential rise in the number of connected devices and continually increasing mobility of these devices. As the number and mobility of the wireless devices increases at a rapid rate leading to HDMANETs, applications that uses OR protocols for communication suffers from three major problems that leads to its transmission inefficiency. Redundant data forwarding at the intermediate devices, high time overhead from frequent packet retransmissions and inefficiency in handling communication voids are the major reasons contributing to this inefficiency and low Quality of Service. Due to these problems, most modern applications that use OR protocols for communication in environments likes volcanoes, hurricane affected regions and underground mines are unable to guarantee excellent Quality of Service and high transmission efficiency to the users.

This research paper proposes Optimized Opportunistic Routing to overcome these problems and to improve the efficiency of opportunistic routing protocols in highly dynamic ad hoc networks. The paper is organized as follows. Section 2 discusses optimized opportunistic routing. 
The performances of the proposed methods are compared with the popular OR protocols using simulations in the next section. Finally, we conclude with future research directions.

\section{Optimized Opportunistic Routing}

A simple and novel opportunistic forwarding technique termed as Optimized Opportunistic Routing (OOR) is proposed. The method would ensure reliable and continuous data transmission between highly mobile devices in HDMANETs and also reduce the time overhead caused by packet retransmissions. The major advantage of OOR is that it is simple and can be easily implemented without major modifications to MAC protocol. Further, the overhead caused is very less compared to existing OR protocols. In OOR every wireless device is assumed to be aware of its position and the position of its immediate neighbor devices. Information about the neighbor devices is piggybacked in the transmitted data packet. The location of the destination device is retrieved by the position look up and registration service given by [13]. As soon as the position of the destination device is obtained, the source device attaches the position information to the data packet. At every forwarder device an inspection is done to check whether the destination device is in its neighbor list. If found the packet is directly delivered to the destination device. This avoids extra routing overhead in our protocol.

Working of OOR is illustrated in Figure 4.1. Two different scenarios are considered. In the first normal scenario, a wireless device S1 wants to send a message to another device 'D1' in the network. Initially device 'S1' creates a candidate list referred as Forwarder Priority List (FPL) of all neighboring devices that are in the transmission range of ' $\mathrm{S} 1$ ' based on the nearness to the destination and using Algorithm 1. The device that is nearest to the destination, device ' $\mathrm{C}$ ' is selected as the Highest Priority Forwarder (HPF) in the list. The remaining devices, Next Forwarder Nodes (NFN) are sorted based on the Expected Distance Advancement with Delivery Probability (EDADP) metric that combines the distance progress to the destination with the probability of data delivery over each transmission link. 


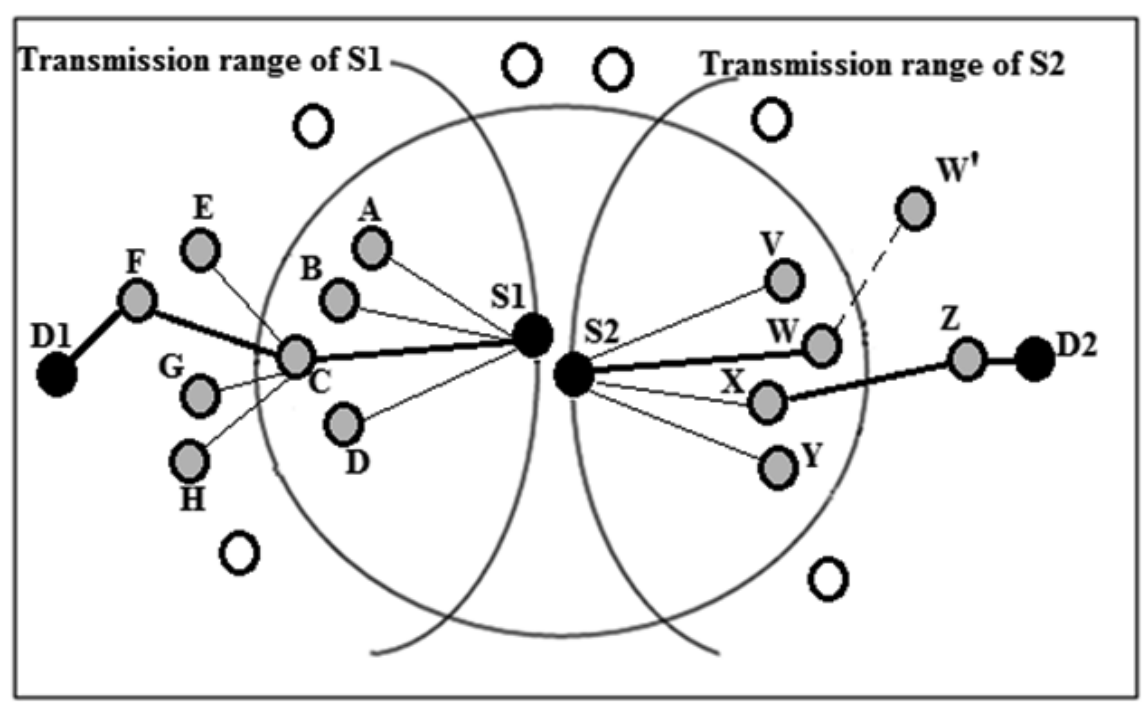

Figure 1. Working of OOR

In all scenarios the device that is nearest to the destination is selected as the Highest Priority Forwarder (HPF). This helps in maximizing the progress of the data packet towards the destination and also reduces the overhead in further computation of other metrics values. Our results show that in majority of cases the data is forwarded by the HPF. Next Forwarder Nodes (NFN) are considered in few cases only. When a data packet is received by the device marked as HPF in the FPL that is attached to the transmitted data packet, it immediately forwards the data packet to the destination. When a data packet is received by all other nodes, they would wait for particular time before retransmission. If it receives a copy of the same data packet within this time, it understands that the HPF has forwarded the data packet and it discards the data packet.

In Scenario 2 a source device 'S2' is transmitting data packets to destination device 'D2' in the network. Here the HPF device ' $W$ ' has moved away from its current location to a new location $\mathrm{W}^{\prime}$ and is unable to forward the data packet. Once the HPF is unable to forward the data packet OOR selects the select the candidate node with maximum EDADP value to forward the data packet to the destination. This ensures minimum retransmissions because the candidate selected has excellent distance progress and very high data delivery probability in the network. So the remaining forwarders (RF) V, X and Y are sorted based on EDADP value nodes. Let us assume that node ' $X$ ' has a higher EDADP value. So if ' $X$ ' doesn't receive a copy of the same data packet within a particular time it understands that HPF has moved away is unable to forward, so ' $\mathrm{X}$ ' 
forwards the data packet. Nodes ' $\mathrm{Y}$ ' and ' $\mathrm{V}$ ' would receive a copy of the same data packet forwarded by ' $\mathrm{X}$ ' and thus discards the data packet and eliminates duplicate forwarding. Expected Distance Advancement with Delivery Probability (EDADP) is calculated using the following equation.

$$
\operatorname{EDADP}\left(\mathrm{S}_{\mathrm{R}}, \mathrm{D}_{\mathrm{T}}, \mathrm{C}_{\mathrm{N}}\right)=\sum_{\mathrm{K}=1}^{\mathrm{N}}\left(\mathrm{D}_{\mathrm{S}_{\mathrm{R}}, \mathrm{D}_{\mathrm{T}}}-\mathrm{D}_{\mathrm{C}_{\mathrm{K}}, \mathrm{D}_{\mathrm{T}}}\right) \times \mathrm{P}_{\mathrm{S}_{\mathrm{K}}, \mathrm{C}_{\mathrm{K}}} \prod_{\mathrm{L}=1}^{\mathrm{K}-1}\left(1-\mathrm{P}_{\mathrm{S}_{\mathrm{R}}, \mathrm{C}_{\mathrm{J}}}\right)
$$

$\mathrm{S}_{\mathrm{R}}$ is the source node, $\mathrm{D}_{\mathrm{T}}$ is the destination node and $\mathrm{C}_{\mathrm{N}}$ is the candidate list of $\mathrm{N}$ probable forwarder nodes. $\left(\mathrm{D}_{\mathrm{S}_{\mathrm{R}}, \mathrm{D}_{\mathrm{T}}}-\mathrm{D}_{\mathrm{C}_{\mathrm{K}}, \mathrm{D}_{\mathrm{T}}}\right)$ gives the expected distance progress of each candidate node to the destination. $\mathrm{P}_{\mathrm{S}_{\mathrm{K}}, \mathrm{C}_{\mathrm{K}}}$ gives the data delivery probability between source node and $\mathrm{K}^{\text {th }}$ candidate node.

Table 4.1 depicts the Forwarder Priority List for both the discussed scenarios in OOR.

Table 2. Forwarder Priority List

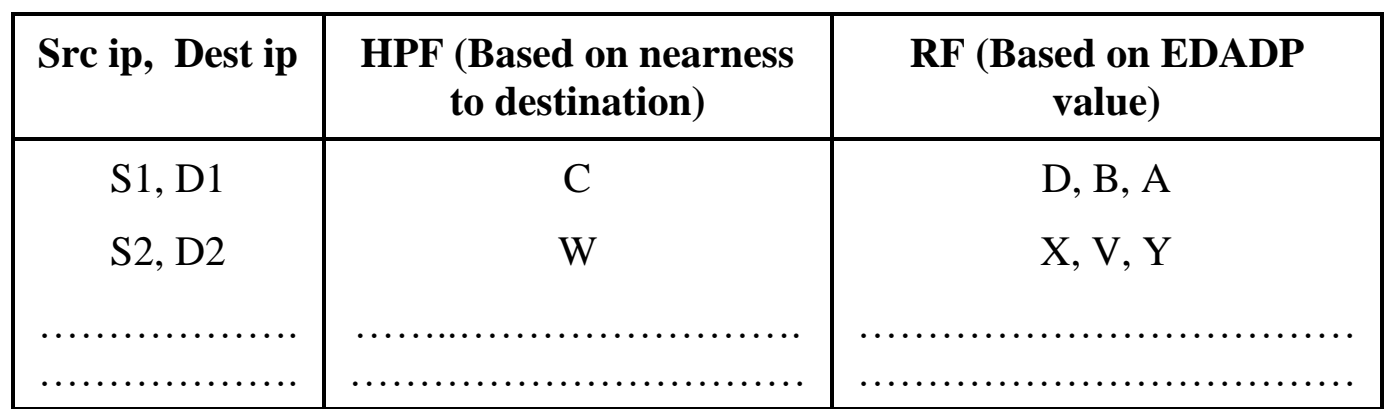

\section{Algorithm1: Constructing the Forwarder Priority List (FPL)}

1. Initialization,

2. set the destination device as $\mathrm{N}_{\mathrm{D}}$,

3. set the Forwarder Priority List as FPL,

4. set the Neighbor Device List as NNL,

5. set the distance from the current device to the destination device $\mathrm{N}_{D}$ as $C_{\text {DIST }}$

6. begin

7. if destination device is in the list of neighbors

8. then 
9. $\quad$ set destination device as the next hop device

10. return

11. end if

12. for $\mathrm{j} \leftarrow 0$ to length(NNL) do

13. NNL[j].dist $\leftarrow \operatorname{dist}\left(\mathrm{NNL}[\mathrm{j}], \mathrm{N}_{\mathrm{D}}\right)$

14. end for

15. NNL.sort()

16. $\mathrm{NNL}[0]=\mathrm{H}_{\mathrm{F}}$

17. $\mathrm{NNL}[1]=\mathrm{M}_{\mathrm{F} 1}$

18. $\mathrm{NNL}[2]=\mathrm{M}_{\mathrm{F} 2}$

19. next hop $\leftarrow \mathrm{H}_{\mathrm{F}}$

20. for $\mathrm{j} \leftarrow 1$ to length(NNL) do

21. if $\operatorname{dist}(\mathrm{NNL}, \mathrm{ND}) \geq$ length of FPL or CDDIST

22. then

23. break

24. else

25. FPL.add(NNL[j])

26. end if

27. end for

Thus using OOR, forwarding of the data packet is ensured as long as there is one device in the FPL leading to high data delivery rate and continuous transmission in the network. Further most of the data is forwarded by the Highest Forwarder Device (HPF) that maximizes the progress of the data packet towards the destination and also reduces the overhead and delay required for further computation. Our results show that in majority of cases the data is forwarded by the HPF. If the HPF is unable to forward the data packet within a particular time the Next Forwarder Node (NFN) is selected to forward the data packet. The NFN is selected based on the highest EDADP value that ensures minimum retransmissions because the candidate node selected has excellent distance progress and very high data delivery probability in the network. Results from Simulations 
with Network Simulator-2 shows that our method achieves very high efficiency and QoS compared to all existing opportunistic routing protocols in HDMANETs.

\section{Performance Analysis}

The performances of the proposed methods are analyzed using Network Siumlator-2 (NS2). Table 4.2 summarizes the parameters used in simulation. The MAC protocol used for the simulation is IEEE $802.11 \mathrm{~g} .100$ nodes are deployed in a network area of $1000 \times 800 \mathrm{~m}^{2}$ rectangular region. The transmission range of the nodes is set at $250 \mathrm{~m}$. Constant Bit Rate (CBR) traffic is being generated from the source to the destination nodes in the network at a rate of 20 packets per second (40kbps). The size of the data packet is set as 512 bytes. The simulation starts at 100 seconds and ends at 900 seconds. Mobility in the network is created by varying the speed of nodes from $5 \mathrm{~m} / \mathrm{s}$ to $50 \mathrm{~m} / \mathrm{s}$ in the network. Random and dynamic movement of nodes is generated using three Random Mobility models, Random Way Point mobility model, Random Walk Mobility Model and Random Direction Mobility Model

Table 3. Simulation Parameters

\begin{tabular}{|l|l|}
\hline MAC Protocol & IEEE $802.11 \mathrm{~g}$ \\
\hline Propagation Model & Two-ray Ground \\
\hline Traffic Type & CBR \\
\hline Network Area & $1000 \times 800 \mathrm{~m}^{2}$ \\
\hline Transmission Range & $250 \mathrm{~m}$ \\
\hline Mobility Model & $\begin{array}{l}\text { Random Way Point Mobility Model } \\
\text { Random Walk Mobility Model } \\
\text { Random Direction Mobility Model }\end{array}$ \\
\hline Size of data packets & $512 \mathrm{bytes}$ \\
\hline Data Rate & $40 \mathrm{kbps}$ \\
\hline Simulation Time & $800 \mathrm{~s}$ \\
\hline Number of devices & 100 \\
\hline $\begin{array}{l}\text { Number of Simulation Runs for each } \\
\text { scenario }\end{array}$ & 10 \\
\hline
\end{tabular}


The performance of the proposed methods is compared with ExOR LCOR [49] and CAOR [48] opportunistic routing protocols. Extremely Opportunistic Routing Protocol (ExOR) is selected for comparison because it's the most referenced opportunistic routing protocol in MANETs. ExOR was one of the first protocols to utilize the opportunistic forwarding mechanism and obtained wide acceptability in opportunistic routing research. Least Cost Opportunistic Routing (LCOR) is selected for comparison because, out of all OR protocols studied; this protocol selects the optimum set of candidate devices in the network and thus has minimum number of retransmissions. Compared to all other OR protocols, this protocol achieves good data delivery in the network with minimum number of retransmissions. Delay experienced by the data packets is also very minimal in this protocol. Thus LCOR is very appropriate for comparison with the proposed methods because it offers higher Quality of Service among all other OR protocols in dynamic MANETs. Context Aware Opportunistic Routing (CAOR) is selected for comparison because it is the latest opportunistic protocol that gives maximum data delivery in the network. Of all the existing OR protocols CAOR delivers maximum number of packets at the destination. CAOR gives very good performance in communication in HDMANETs compared to other opportunistic routing protocols.

\section{Results and Discussion}
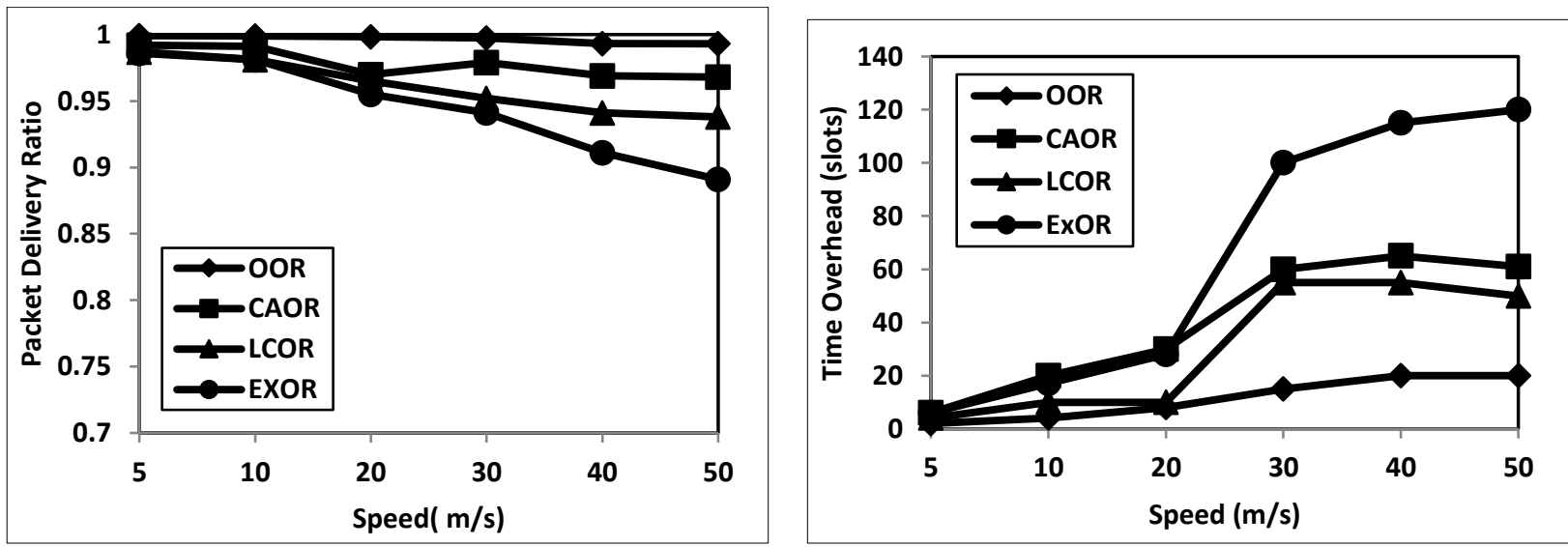

a. PDR vs Speed

b. Time Overhead vs Speed 


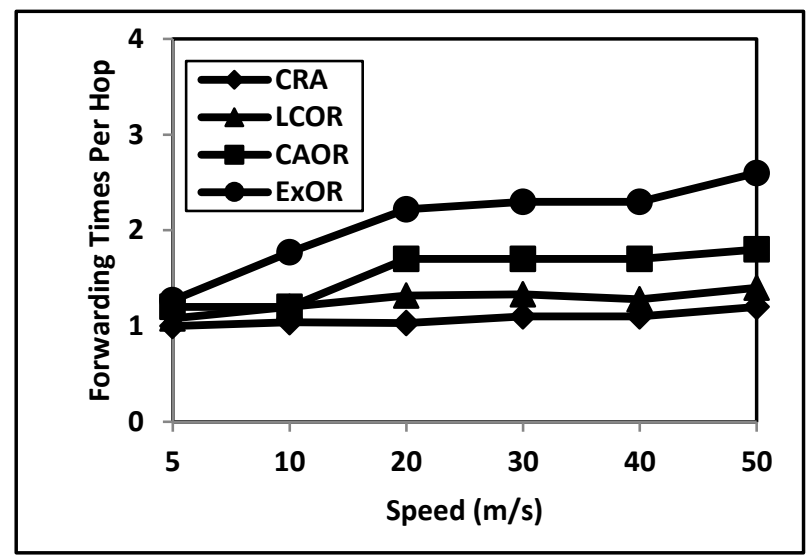

c. FTPH vs Speed

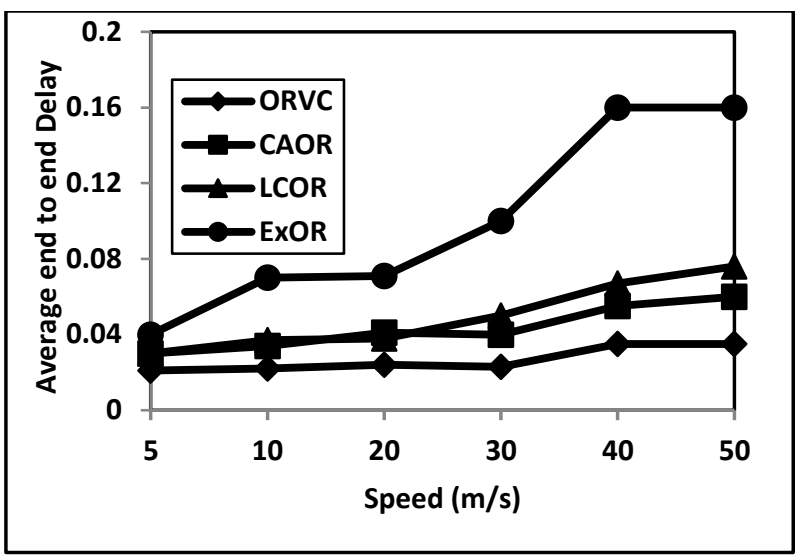

d. Average end to end delay vs

Speed

Figure 4. Simulation Results with Random Way Point Mobility Model

Figure 4 gives the performance comparison of protocols with the random way point mobility model. It is very evident that the proposed methods offer better performance to all the existing opportunistic routing protocols in highly dynamic ad hoc networks. The same could be interpreted from figure 5 and figure 6 which displays results with random walk and random direction mobility models respectively. 


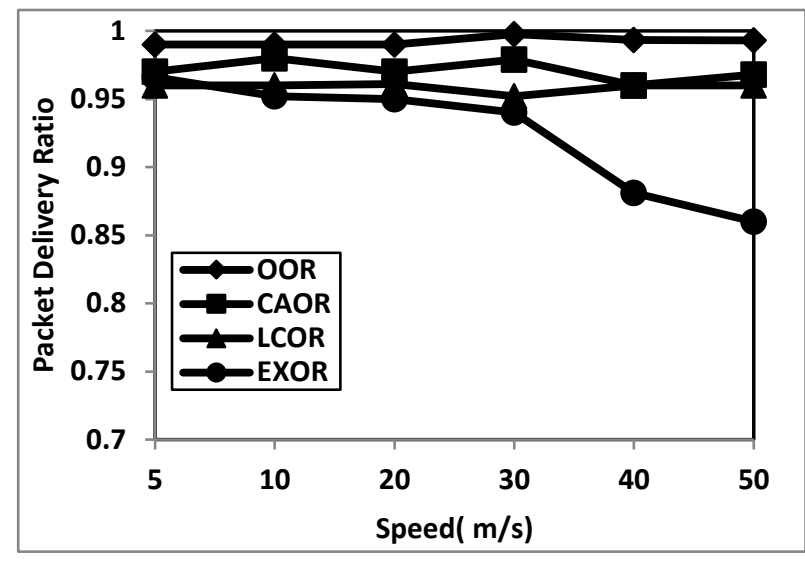

a. PDR vs Speed

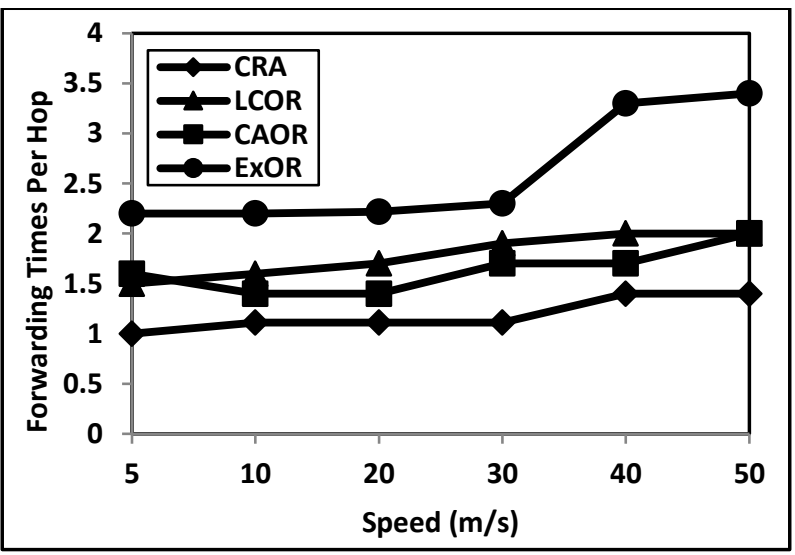

c. FTPH vs Speed

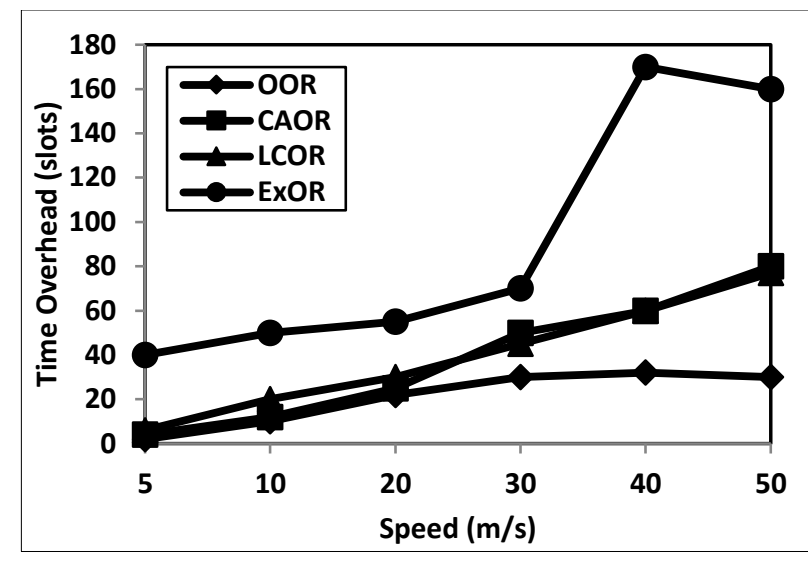

b. Time Overhead vs Speed

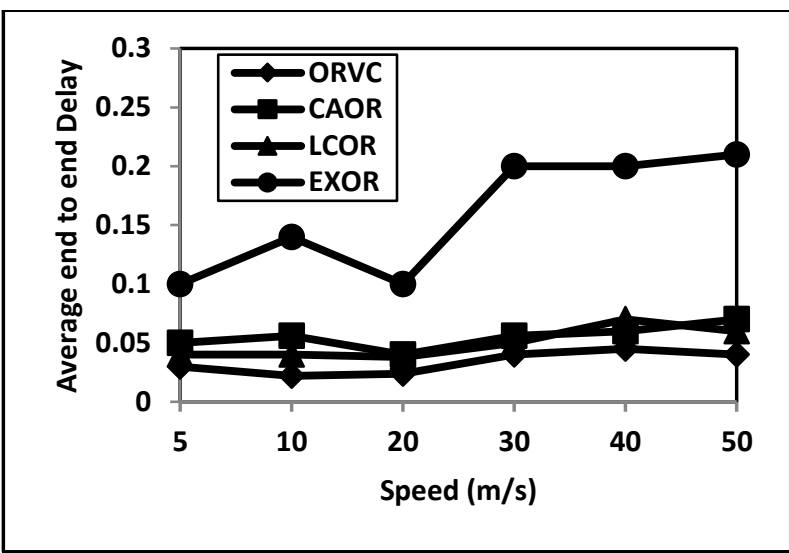

d. Average end to end delay vs

Speed

Figure 5. Simulation Results with Random Walk Mobility Model 


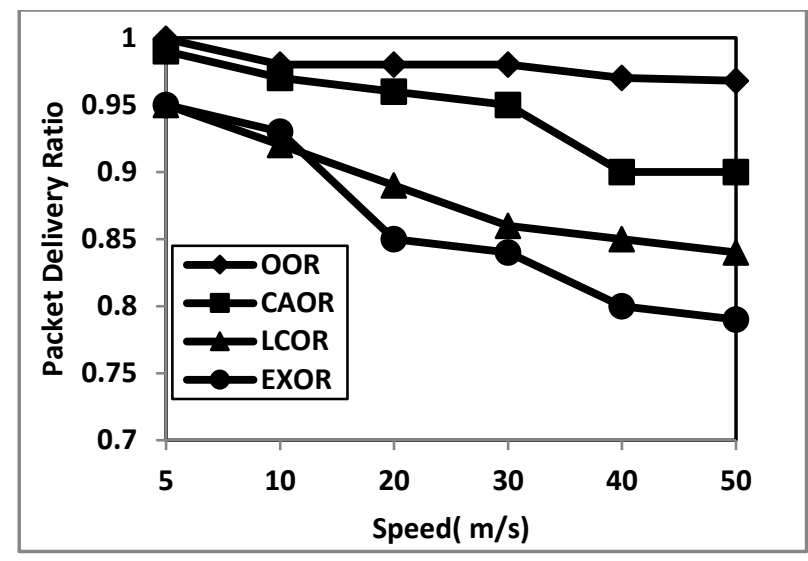

a. PDR vs Speed

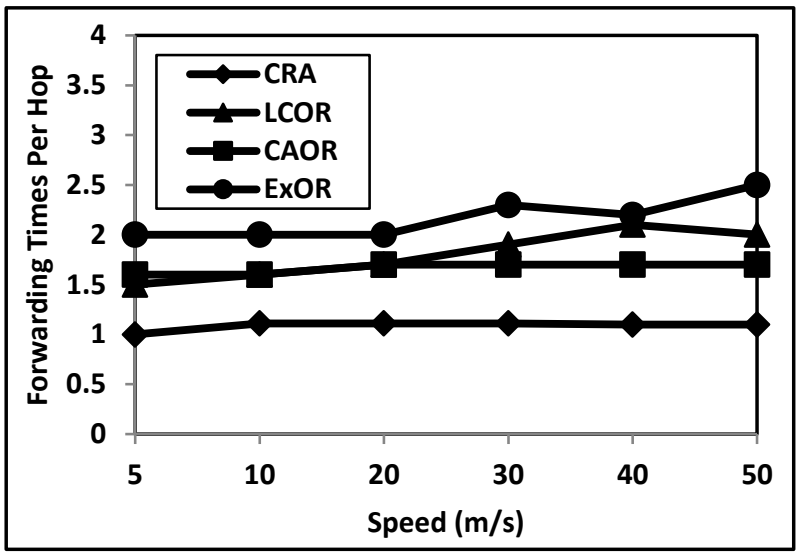

c. FTTH vs Speed

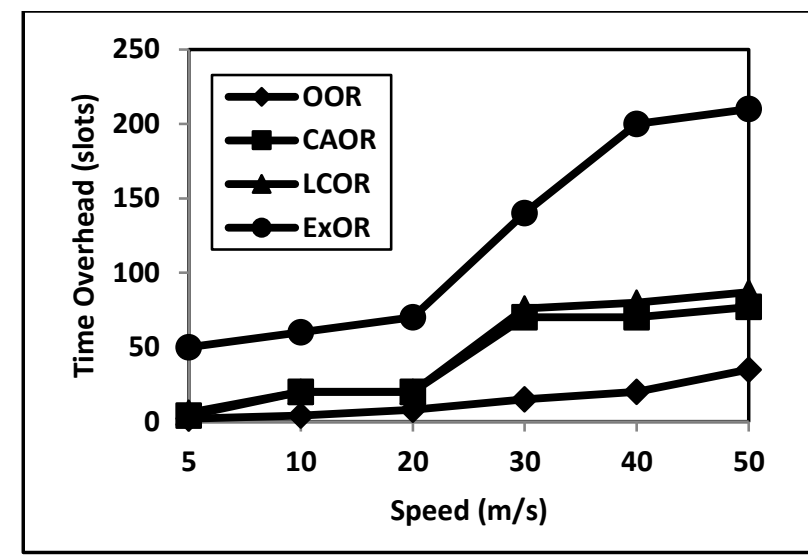

b. Time Overhead vs Speed

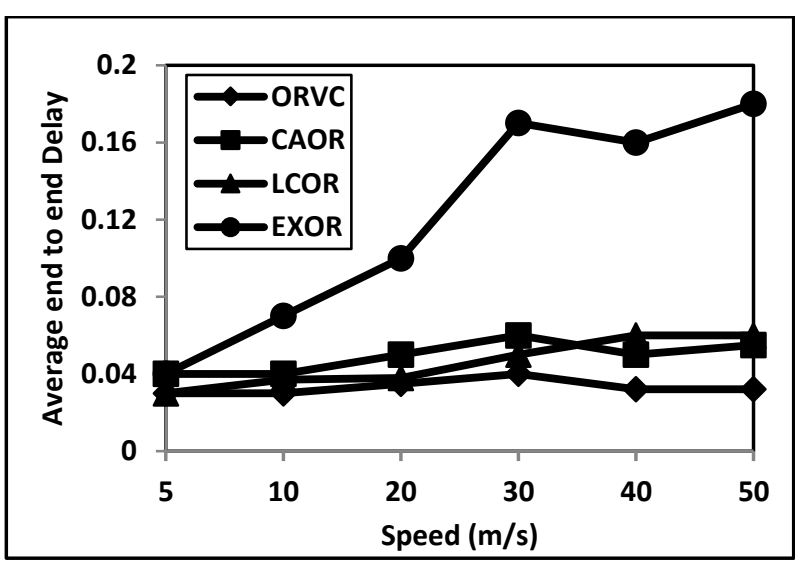

d. Average end to end delay vs

Speed

Figure 6. Simulation Results with Random Direction Mobility Model

\section{Conclusion and Future Research Directions}

As the speed and mobility of devices increases in the network, performances of the existing opportunistic routing protocols degrade considerably, leading to reduced Quality of Service and poor transmission efficiency. The exceptional potential of opportunistic routing is thus underutilized. Redundant data forwarding at the intermediate nodes, high time overhead from frequent packet retransmissions and inefficiency in handling communication voids are the 
reasons contributing to this transmission inefficiency. The paper proposed Optimized Opportunistic Routing (OOR) strategy that guarantee excellent Quality of Service and high transmission efficiency to the latest applications using opportunistic routing for communication in highly dynamic ad hoc networks. Simulation results showed that our method achieve significant performance improvements compared to all other existing opportunistic routing protocols in highly dynamic mobile ad hoc networks. Further these methods could also be implemented for routing in Internet of Things [42-44] underwater swarm robots [45] and vehicular fog networks [46-47].

\section{References}

[1] S. Giordano and W. Lu, Challenges in Mobile Ad Hoc Networking, IEEE Communications Magazine, 39(6) (2001)129-129.

[2] I. Chlamtac, M. Conti and J. Liu, Mobile Ad Hoc Networking: Imperatives and Challenges, Ad Hoc Networks, 1(1) (2003) 13-64.

[3] E. Gelenbe, Quality of Service in Ad Hoc Networks, Ad Hoc Networks, 2(3) (2004) 203204.

[4] J. Hoebeke, I. Moerman, B. Dhoedt and P. Demeester, An Overview of Mobile Ad Hoc Networks: Applications and Challenges, Journal of The Communications Network, 3(3) (2004) pp.60-66.

[5] M. Conti, and S. Giordano, Mobile Ad Hoc Networking: Milestones, Challenges, and New Research Directions, IEEE Communications Magazine, 52(1) (2014) 85-96.

[6] Varun G. Menon and Joe Prathap P. M., Performance of various Routing Protocols in Mobile A Hoc Networks-A Survey, Research Journal of Applied Sciences, Engineering and Technology, 6(22), pp. 4181-4185, December 2013.

[7] Varun G Menon and Joe Prathap P M, "Routing in Highly Dynamic Ad Hoc Networks: Issues and Challenges", International Journal of Computer Science and Engineering, vol.8, no. 4, pp.112-116, 2016.

[8] Varun G Menon, Sreekala C S, Vibin J, Teenu T. ,Eldho A, "Performance Analysis of Traditional Topology based Routing Protocols in Mobile Ad hoc Networks"The International Journal of Computer Science Applications, vol. 2,no.1, pp. 1-6. 
[9] Varun G Menon \& Joe Prathap, P. M. (2017). Towards Optimal Data Delivery in Highly Mobile Wireless Ad Hoc Networks. International Journal of Computer Science and Engineering, 9(1), 1-6.

[10] Varun G Menon "Analyzing the Performance of Random Mobility Models with Opportunistic Routing." Volume 10, Number 5 (2017), pp. 1221-1226

[11] Perkins, C. E. and Bhagwat, P. (1994), "Highly Dynamic Destination-Sequenced DistanceVector Routing (DSDV) for Mobile Computers", Proceedings of the Conference on Communications Architectures, Protocols and Applications (SIGCOMM '94). ACM, New York, USA, pp. 234-244.

[12] Jacquet, P., Muhlethaler, P. and Qayyum, A. (1998), "Optimized Link State Routing Protocol", Internet Draft, Available at draft-ietf-manetolsr-00.txt.

[13] Bellur, B., Ogier, R.G. and Templin, F.L. (2001), “Topology Broadcast Based on ReversePath Forwarding (TBRPF)", IETF Internet Draft, Available at draft-ietf-manet-tbrpf01.txt.

[14] Johnson, D. B. and Maltz, D. A. (1996), "Dynamic Source Routing in Ad Hoc Wireless Networks", Mobile Computing, Kluwer Academic Publishers, vol. 353, Chapter 5, pp. 153-181.

[15] Toh, C-K. (1996), “A Novel Distributed Routing Protocol to Support Ad-Hoc Mobile Computing", Conference Proceedings of the 1996 IEEE Fifteenth Annual International Phoenix Conference on Computers and Communications, Scottsdale, AZ, 1996, pp. 480486.

[16] Perkins, C. E. and Royer, E. M. (1999), “Ad-Hoc On-Demand Distance Vector Routing”, Proceedings of Second IEEE Workshop on Mobile Computing Systems and Applications, New Orleans, USA, pp. 90-100.

[17] Park V. D. and Corson, M. S. (1997), “A Highly Adaptive Distributed Routing Algorithm for Mobile Wireless Networks", Proceedings of the Sixteenth Annual Joint Conference of the IEEE Computer and Communications Societies, Kobe, pp. 1405-1413.

[18] Karp, B. and Kung, H. T. (2000), “GPSR: Greedy Perimeter Stateless Routing for Wireless Networks", Proceedings of The 6th Annual International Conference on Mobile Computing and Networking (MobiCom '00), ACM, New York, USA, pp. 243-254.

[19] Mauve, M., Widmer, J., and Hartenstein, H. (2001), “A Survey on Position-Based Routing in Mobile Ad Hoc Networks”, IEEE Network, vol. 15, no.6, pp. 30-39.

[20] Cadger, F., Curran, K., Santos J and Moffett, S (2013), “A Survey of Geographical Routing in Wireless Ad-Hoc Networks", IEEE Communications Surveys and Tutorials, vol. 15, no. 2, pp. 621-653. 
[21] Varun G Menon, Jogi Priya P M, Joe Prathap P M, "Analyzing the behavior and performance of greedy perimeter stateless routing protocol in highly dynamic mobile ad hoc networks", Life Science Journal , 10(2): pp 1601-1605, 2013.

[22] Varun Menon and Joe Prathap P.M, "Performance analysis of geographic routing protocols in highly mobile ad hoc network," Journal of Theoretical and Applied Information Technology, vol. 54 no. 1, pp 127-133, 2013.

[23] Biswas,S. and Morris,R. (2005), "ExOR: Opportunistic Multi-Hop Routing for Wireless Networks", Proceedings of the 2005 Conference on Applications, Technologies, Architectures, and Protocols for Computer Communications (SIGCOMM '05), ACM, New York, USA, pp. 133-144.

[24] Bruno,R., Conti, M. and Nurchis, M. (2010), "Opportunistic Packet Scheduling and Routing in Wireless Mesh Networks", Proceedings of IFIP Wireless Days Conference, Venice, Italy, pp. 1-6.

[25] Chakchouk, N. (2015), "A Survey on Opportunistic Routing in Wireless Communication Networks”, IEEE Communications Surveys and Tutorials, vol. 17, no. 4, pp. 2214-2241.

[26] Menon V. G. and Joe Prathap P M, (2016), "Comparative Analysis of Opportunistic Routing Protocols for Underwater Acoustic Sensor Networks" Proceedings of the IEEE International Conference on Emerging Technological Trends, Kerala, India.

[27] Menon V G, “Opportunistic Routing Protocols in Underwater Acoustic Sensor Networks: Issues, Challenges, and Future Directions", Magnetic Communications: From Theory to Practice, CRC Press, pp. 127-148, 2018

[28] Menon V G and Joe Prathap PM, "Moving From Topology-Dependent to Opportunistic Routing Protocols in Dynamic Wireless Ad Hoc Networks: Challenges and Future Directions", Algorithms, Methods, and Applications in Mobile Computing and Communications, IGI Global, 1-23

[29] Menon V G and Joe Prathap P M, "A Review on Efficient Opportunistic Forwarding Techniques used to Handle Communication Voids in Underwater Wireless Sensor Networks, Advances in Wireless and Mobile Communications, vol 10, no. 5, pp. 10591066, 2017

[30] Varun G Menon and Joe Prathap P.M, "Analysing the Behaviour and Performance of Opportunistic Routing Protocols in Highly Mobile Wireless Ad Hoc Networks", International Journal of Engineering and Technology, vol. 8, no. 5, pp. 19161924, 2016.

[31] Varun G Menon, Joe Prathap P M and Vijay A, "Eliminating Redundant Relaying of Data Packets for Efficient Opportunistic Routing in Dynamic Wireless Ad Hoc Networks",Asian Journal of Information Technology,vol. 12, no.17, 2016 
[32] Varun G Menon, "Survey on Latest Energy Based Routing Protocols for Underwater Wireless Sensor Networks", International Journal of Computer Networks and Wireless Communications (IJCNWC), Vol.6, No 6, Nov-Dec 2016, pp-52-55.

[33] V. G. Menon and P. M. Joe Prathap, "Opportunistic routing with virtual coordinates to handle communication voids in mobile ad hoc networks," in Advances in Signal Processing and Intelligent Recognition Systems, vol. 425 of Advances in Intelligent Systems and Computing, pp. 323-334, Springer International, 2016.

[34] Varun G. Menon, Joe Prathap Pathrose, and Jogi Priya, "Ensuring Reliable Communication in Disaster Recovery Operations with Reliable Routing Technique,” Mobile Information Systems, vol. 2016, Article ID 9141329, 10 pages, 2016.

[35] Mase, K. (2011), "How to Deliver Your Message from/to a Disaster Area", IEEE Communications Magazine, vol. 49, no. 1, pp. 52-57.

[36] Cadger, F., Curran, K., Santos, J. and Moffett, S. (2016), "Location and Mobility-Aware Routing for Multimedia Streaming in Disaster Telemedicine", Ad Hoc Networks, vol. 36, no.1, pp. 332-348.

[37] Leiner, B. M., Ruther, R. J. and Sastry, A. R. (1996), "Goals and Challenges of the DARPA Glomo Program [Global Mobile Information Systems]", IEEE Personal Communication, vol.3, no.6, pp. 34-43.

[38] Yoon, S. G., Jang, S., Kim, Y. H. and Bahk, S. (2014), "Opportunistic Routing for Smart Grid with Power Line Communication Access Networks", IEEE Transactions on Smart Grid, vol. 5, no.1, pp. 303-311.

[39] Gorbil, G. and Gelenbe, E. (2012), "Resilience and Security of Opportunistic Communications for Emergency Evacuation", Proceedings of the 7th ACM Workshop on Performance Monitoring and Measurement of Heterogeneous Wireless and Wired Networks, New York, USA, pp.115-124.

[40] Martin-Campillo, A., Crowcroft, J., Yoneki, E. and Marti, R. (2013), "Evaluating Opportunistic Networks in Disaster Scenarios", Journal of Network and Computer Applications, vol. 36, no. 2, pp. 870-880.

[41] Pentland, A., Fletcher, R. and Hasson, A. (2004), "DAKNET: Rethinking Connectivity in Developing Nations", IEEE Computer Magazine, vol. 37, no. 1, pp. 78-83.

[42] Deshkar, S., Thanseeh, R. A., \& Menon, V. G. (2017). A Review on IoT based m-Health Systems for Diabetes. International Journal of Computer Science and Telecommunications, 8(1), 13-18.

[43] Philip, V., Suman, V. K., Menon, V. G., \& Dhanya, K. A. (2017). A Review on latest Internet of Things based Healthcare Applications. International Journal of Computer Science and Information Security, 15(1), 248. 
[44] Vinoj, P. G., Jacob, S., \& Menon, V. G. (2018, September). Hybrid brainactuated muscle interface for the physically disabled. In BASIC \& CLINICAL PHARMACOLOGY \& TOXICOLOGY (Vol. 123, pp. 8-9). 111 RIVER ST, HOBOKEN 07030-5774, NJ USA: WILEY.

[45] K S, Keerthi \& Mahapatra, Bandana \& G Menon, Varun. (2018). Into the World of Underwater Swarm Robotics: Architecture, Communication, Applications and Challenges. Recent Patents on Computer Science. 12.

[46] Menon, Varun G., and Joe Prathap. "Vehicular Fog Computing: Challenges Applications and Future Directions." International Journal of Vehicular Telematics and Infotainment Systems (IJVTIS) 1.2 (2017): 15-23.

[47] Menon V G, Moving From Vehicular Cloud Computing to Vehicular Fog Computing: Issues and Challenges International Journal on Computer Science and Engineering, vol. 9, no. 2, pp. 14-18, 2017.

[48] Zhao, Z., Rosario, D., Braun, T., Cerqueira, E., (2014), "Context-Aware Opportunistic Routing in Mobile Ad-Hoc Networks Incorporating Device Mobility", Proceedings of the IEEE Wireless Communications and Networking Conference, Istambul, Turkey, pp. 21382143.

[49] Dubois-Ferriere, H., Grossglauser, M. and Vetterli, M. (2007), "Least-Cost Opportunistic Routing", Proceedings of 2007 Allerton Conference on Communication, Control, and Computing, Allerton, UK, pp. 1-8. 